\title{
Clinical pattern of systemic sclerosis in Central Ukraine. Association between clinical manifestations of systemic sclerosis and hypertension
}

\author{
Viktor Semenov ${ }^{1}$, Olexandr Kuryata ${ }^{1}$, Tatiana Lysunets ${ }^{2}$ \\ ${ }^{1}$ Internal Medicine 2, State Establishment "Dnipropetrovsk Medical Academy of Health Ministry of Ukraine" \\ ${ }^{2}$ Rheumatology Department, Municipal institution "Mechnikov Dnipropetrovsk regional clinic", Dnipro, Ukraine
}

\begin{abstract}
Objectives: Systemic sclerosis (SSc) is a rare disease of connective tissue, manifestations of which may vary in different geographical areas. We aimed to describe the clinical portrait of patients with SSc in Dnipropetrovsk region and to investigate how initial clinical and laboratory characteristics are connected with the presence of hypertension in SSc onset.

Material and methods: Patients were enrolled to this study from the registry of SSc patients, established in the Rheumatology Department, Mechnikov Dnipropetrovsk Regional Clinic, Dnipro. This registry contains histories of new cases of SSc from 1993 to 2014. Patients are followed-up and receive treatment according to EULAR and local standards. Diagnosis of SSc was based on ACR and EULAR Criteria for systemic Sclerosis. Two patients developed scleroderma renal crisis during follow-up. This report is a cross-sectional study. We analysed only data of the first visit to a rheumatologist.

Results: In total 148 patients (median age [IQR] - 47 [40; 52] years) fulfilled the inclusion criteria. Male/female ratio was $1: 20.1$. The most frequent clinical signs were Raynaud's phenomenon and arthritis. The prevalence of skin lesion in dcSSc patients was twice as high as in IcSSc patients. Pulmonary fibrosis occurred significantly more commonly in dcSSc patients. Hypertension occurred in 26-33\% in both groups. Patients with hypertension at the SSc onset were seven years older than normotensive patients. More hypertensive patients were classified as IcSSc. Mean GFR was dramatically lower in hypertensive patients.

Conclusions: The most common clinical form in our study was diffuse cutaneous subset of SSc. Hypertension in patients with SSc may be associated with local cutaneous subset of SSc and renal impairment. The strongest predictors of clinical form of SSc are signs of fibrosis (skin lesion and pulmonary fibrosis) and inflammation (arthritis and elevated CRP).
\end{abstract}

Key words: systemic sclerosis, registry, arterial hypertension.

\section{Introduction}

Systemic sclerosis (SSc) is a rare disease of connective tissue that may affect different organs and is characterised by immune, vascular, and fibrotic disruptions. Because of the low prevalence of SSc in the population, providing clinical trials and wide observations may be challenging. To overcome the lack of reliable epidemiological data, different medical and scientific communi- ties have established registries to collect and analyse data about SSc. Investigating the patient population is important because the incidence, prevalence, and clinical features of SSc throughout the world differ.

Epidemiological data from South Australia show an incidence rate of SSc 22.8 (95\% Cl) of new cases per million [1] (data from 1993-1999), while in Greece the incidence rate is 11 new cases per million (data from

Address for correspondence:

Viktor Semenov, Internal Medicine 2, State Establishment “Dnipropetrovsk Medical Academy of Health Ministry of Ukraine”, Ukraine, e-mail: kinsolwing@gmail.com

Submitted: 24.01.2018; Accepted: 23.02.2018 
1981-2002) [2]. Differences in the assessment of incidence can be explained by different ethnic and genetic compositions of different regions, climatic conditions, exposures, etc. [3, 4].

Analysis of registries' data allows us to determine the factors that can impact prognosis of the disease. For example, data from the EULAR registry have shown that deterioration of skin fibrosis can be predicted by initial modified Rodnan skin score [5]. In the DUO registry several factors were revealed, capable of predicting development of incident gangrene [6].

The high heterogeneity of clinical manifestations has prompted internists to classify SSc to facilitate its management. In 1980 the American College of Rheumatology presented the Systemic Sclerosis Classification Criteria, which were précised in 1988, 2001, and 2013 [7-10]. Now we have highly specific and sensitive criteria that allow us to distinguish different forms of SSc. But still there are some patients who cannot be classified. Using registries helps rheumatologists to find symptoms that are useful in distinguishing subsets. Minier et al., in research based on the EUSTAR registry, declared puffy fingers to be a pivotal sign for suspicion of systemic sclerosis [11]. In our investigation we aimed to describe the clinical portrait of patients with SSc in the Dnipropetrovsk region (central Ukraine). This region is characterised by multinational composition. Officially, $80 \%$ of the population of the region are Ukrainians, but considering the complicated history of Ukraine (frequent changes of state power and migration) we assume a high diversity of nationalities represented here.

In our previous studies we learned that hypertension in SSc patients has heterogeneous aetiology and can be predicted by age $>45$ years, presence of skin lesions, and elevated markers of inflammation [12]. But still there is a lack of information about the role of hypertension in progression of SSc. We know about the prevalence of hypertension on the onset of SSc $[13,14]$. Patients with rheumatoid arthritis and inflammatory joint diseases have higher cardiovascular risk, compared to the general population. There are recommendations for cardiovascular risk management in this group of patients issued by EULAR [15]. These recommendations could be used for SSc patients also, but we assume that because of the specific genesis of scleroderma, such patients require a separate approach.

It is known that hypertension can cause vascular changes similar to those that occur in SSc [16], and probably these two conditions may interact with each other. Also, interaction between hypertension and SSc-related vascular damage may result in a separate phenotype of hypertension in such patients. This thesis is fair at least for SSc-related pulmonary hypertension (PAH) - recent studies have shown that idiopathic PAH is significantly histologically different from SSc-related pulmonary hypertension [17]. There are suggestions that SSc-related renal changes can be histologically differentiated from those in hypertension [18]. Although there are data indicating that vascular changes in SSc patients are primary to hypertension rather than secondary, they lack evidence $[19,20]$. In this study we aimed to investigate how initial clinical and laboratory characteristics are connected with the presence of hypertension in SSc onset.

\section{Material and methods}

Patients were enrolled to this study from the registry of SSc patients established in the Rheumatology Department, Mechnikov Dnipropetrovsk Regional Clinic, Dnipro. This registry contains histories of new cases of SSc since 1993. Patients are followed-up and receive treatment according to EULAR [21] and local standards. Diagnosis of SSc was based on ACR (including LeRoy and Medsger modifications) and EULAR Criteria for Systemic Sclerosis [7-10]. This report is cross-sectional study. We analysed only data of the first visit to a rheumatologist from 1993 to 2014. Two patients developed scleroderma renal crisis (SRC) during follow-up and were excluded from analysis (Fig. 1). All patients gave written, informed consent; the study was approved by the Ethics Committee at the Mechnikov Dnipropetrovsk Regional Clinic.

Patients were classified according to LeRoy and Medsger's modifications of ACR Criteria $[8,9]$ into the following groups: limited SSc (pre-SSc) [22], limited cutaneous SSc (IcSSc), diffuse cutaneous SSc (dcSSc), and "other" (patients with localised forms of SSc and overlap-syndrome were included into this group). Limited SSc was defined as the presence of Raynaud's phenomenon (objective) plus any one of the following: SSc-type

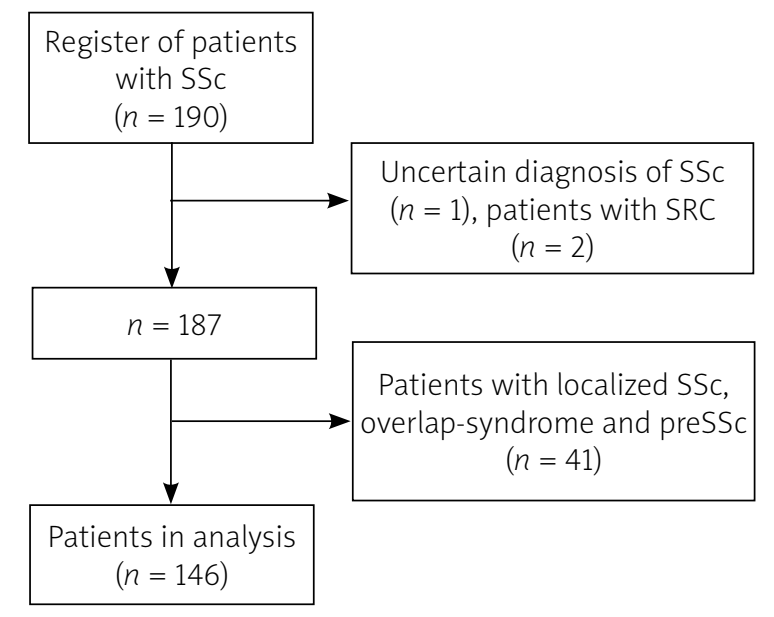

Fig. 1. Study design. 
nailfold capillary pattern or SSc selective autoantibodies; or Raynaud's phenomenon (subjective only) plus both SSc-type nailfold capillary pattern and SSc selective autoantibodies. Limited cutaneous subset was defined in patients with criteria for pre-SSc plus distal cutaneous changes. Diffuse cutaneous subset was defined in patients with criteria for pre-SSc plus proximal cutaneous changes. Patients with pre-SSc, localised forms, and overlap-syndrome were excluded from the analysis because of heterogeneity of this group and the absence of a clear definition of pre-SSc.

Raynaud's phenomenon was defined using clinical (development of the paleness of skin of the fingers in response to cold exposure or emotional stress) and nailfold video capillaroscopy. Skin lesion was defined if a patient had focal swelling, induration, or atrophy, regardless of localisation. Arthritis was regarded as swelling and tenderness in one or more joints. Presence of lung fibrosis was assessed using chest X-ray or CT examination. Gastrointestinal involvement was diagnosed in the case of complaints of dysphagia accompanied by changes on gastroscopy or barium swallow test. Hypertension was diagnosed using local standards and 2013 ESH/ESC Guidelines for the management of arterial hypertension [23].

Anaemia was diagnosed as a haemoglobin level $>130 \mathrm{~g} / \mathrm{l}$ in males and > $120 \mathrm{~g} / \mathrm{l}$ in females. The level of leukocytes was regarded abnormal if it was higher than $9 \times 10^{9} /$ or lower than $4 \times 10^{9} /$. The level of thrombocytes was regarded as abnormal if it was higher than $320 \times$ $10^{9} /$ or lower than $180 \times 10^{9} / \mathrm{l}$. We used a cut-off point of $25 \mathrm{~mm} /$ hour for erythrocyte sedimentation rate (ESR) because of the proven impact of that value on the prognosis [24]. Glomerular filtration rate (GFR) was estimated using the CKD-EPI formula [25]. For detection of the proteinuria we used urine microelectrophoresis (> 0.3 and $<3.5 \mathrm{~g}$ ) 24 hours according to local standards). We used standard methods of measurement for C-reactive protein (CRP) and rheumatoid factor (RF). Mean pressure in pulmonary artery (PAPm) was assessed during Doppler echocardiogram. In multivariate logistic regression we used proteinuria as a marker of vascular damage [26].

The data was the most complete for age, gender clinical manifestations (Raynaud's phenomenon, arthritis, skin lesion, pulmonary fibrosis, hypertension), anaemia, leukocyte count, ESR, cylindruria, and proteinuria with missing data for the mentioned values $<10 \%$. The percentage of missing data for the rest of the variables ranged between 20 and 50\%, being the highest for GFR (70.3\%). The percentage presented in the tables along with absolute values reflects valid proportion.

Data were analysed using Microsoft Office and SPSS 17.0. The type of data distribution was assessed with
Kolmogorov-Smirnov test, and differences in variances of compared groups were assessed with Levene's test. Normally distributed data were described with mean and standard deviation (SD), and non-normally distributed data were described with median and interquartile range (IQR). Data with normal distribution and equal variance were compared using independent T-test. In the case of violation of assumptions of data distribution, normality or homogeneity of variance U-Mann-Whitney was performed. We used two-tailed Fisher's exact test to compare categorical data. Investigation of predictors of clinical forms was performed using multivariate logistic regression with stepwise forward method of entering variables. Predictors in the model were: symptoms of excessive fibrosis (skin lesion, pulmonary fibrosis), signs of vasculopathy (hypertension, proteinuria), and signs of inflammation (arthritis, elevated levels of CRP, RF or ESR). We considered $p$-values $<0.05$ to be significant.

\section{Results}

We analysed data of 148 patients (seven males and 139 females, median age [IQR] 47 [40; 52] years). Male/ female ratio was $1: 19.8$. Males in our study contributed only to the dcSSc group (Table I).

The median age of the disease onset in our study was slightly higher in IcSSc compared to dcSSc patients. The most frequent clinical signs were Raynaud's phenomenon and arthritis. Prevalence of RP and arthritis was high in both groups. Prevalence of skin lesion in dcSSc patients was twice as high as in IcSSc patients $(p<0.001)$. Pulmonary fibrosis occurred significantly more often in dcSSc than in IcSSc patients $(p=0.01)$. The prevalence of gastrointestinal complications was slightly higher in patients with IcSSc. Hypertension occurred in $26-33 \%$ in both groups. Among laboratory signs we observed higher prevalence of anaemia, elevated CRP $(p=0.03)$, proteinuria, and cylindruria in the dcSSc group. Mean GFR was higher IcSSc. PAPm was normal in both groups (Table I).

Patients who had hypertension at SSc onset were seven years older than normotensive patients. More hypertensive patients were classified as ICSSc, but not significantly. Hypertension at SSc onset was associated with higher prevalence of skin lesion, arthritis, pulmonary fibrosis, abnormal levels of thrombocytes and ESR, and cylindruria. The prevalence of gastrointestinal complications in hypertensive patients was higher. The absence of hypertension was associated with higher prevalence of anaemia. In hypertensive patients PAPm was slightly higher and mean GFR was dramatically lower (Table II).

We aimed do distinct clinical and laboratory signs of SSc at the disease onset that could help rheumatologist 
Table I. General clinical and laboratory characteristics

\begin{tabular}{|c|c|c|c|c|}
\hline \multirow[t]{2}{*}{ Patient characteristics } & Total & IcSSC & $\mathrm{dcSSc}$ & $p$-value \\
\hline & \multicolumn{4}{|c|}{$n$ (valid \%) } \\
\hline Number of patients & $\begin{array}{c}146 \\
(100) \\
\end{array}$ & $\begin{array}{c}39 \\
(26.8) \\
\end{array}$ & $\begin{array}{c}107 \\
(73.2) \\
\end{array}$ & \\
\hline Median age, years [IQR] & $47[40 ; 52]$ & $48[41 ; 51]$ & $46[40 ; 52]$ & 0.74 \\
\hline Female & $139(95.2)$ & $39(100.0)$ & $100(93.5)$ & 0.12 \\
\hline Male & $7(4.8)$ & $0(0.0)$ & $7(6.5)$ & 0.12 \\
\hline Raynaud's phenomenon & $127(92.7)$ & $35(94.6)$ & $92(92.0)$ & 0.72 \\
\hline Skin lesion & $90(61.6)$ & $14(35.9)$ & $76(71.0)$ & $<0.001$ \\
\hline Arthritis & $120(82.2)$ & $34(87.2)$ & $86(80.4)$ & 0.46 \\
\hline Pulmonary fibrosis & $37(25.3)$ & $4(10.3)$ & $33(30.8)$ & 0.01 \\
\hline Gastrointestinal involvement & $77(65.8)$ & $18(75.0)$ & $59(63.4)$ & 0.28 \\
\hline Oesophagus* & $52(54.7)$ & $15(58.8)$ & $37(52.8)$ & 0.53 \\
\hline Hypertension & $39(26.7)$ & $12(30.8)$ & $27(25.2)$ & 0.53 \\
\hline Anaemia & $51(35.4)$ & $10(25.6)$ & $41(39.0)$ & 0.17 \\
\hline Abnormal leukocytes & $27(19.1)$ & $5(12.8)$ & $22(21.6)$ & 0.33 \\
\hline Abnormal thrombocytes & $25(24.3)$ & $6(22.2)$ & $19(25.0)$ & 1.0 \\
\hline $\mathrm{ESR}>25 \mathrm{~mm} / \mathrm{h}$ & $28(20.4)$ & $6(16.2)$ & $22(22.0)$ & 0.63 \\
\hline $\mathrm{CRP}>10 \mathrm{mg} / \mathrm{l}$ & $22(19.1)$ & $2(6.3)$ & $20(24.1)$ & 0.03 \\
\hline $\mathrm{RF}>14 \mathrm{IU} / \mathrm{ml}$ & $10(10.1)$ & $1(3.3)$ & $9(13.0)$ & 0.27 \\
\hline Proteinuria & $43(31.4)$ & $10(27.0)$ & $33(33.0)$ & 0.54 \\
\hline Cylindruria & $14(10.6)$ & $3(8.6)$ & $11(11.3)$ & 0.76 \\
\hline Mean GFR, ml/min (SD) & $88.5(22.9)$ & $93.7(23.1)$ & $88.0(23.1)$ & 0.63 \\
\hline Median PAPm, mm Hg [IQR] & $23[17 ; 32]$ & $23[16 ; 32]$ & $23[17 ; 32]$ & 0.77 \\
\hline
\end{tabular}

ICSSC - limited cutaneous subset of SSC; dcSSc - diffuse cutaneous subset of SSC; ESR - erythrocyte sedimentation rate; CRP - C-reactive protein; RF-rheumatoid factor; GFR - glomerular filtration rate; PAPm - mean pulmonary arterial pressure; IQR - interquartile range; $S D$ - standard deviation

* Oesophageal involvement was isolated from the manifestation of the digestive system as an important risk factor for poor prognosis.

to predict the form of SSc. We used parameters from the registry that had the lowest levels of missing data and distributed them into the following groups: markers of inflammation (arthritis, elevated CRP, ESR, and RF); markers of fibrosis (pulmonary fibrosis, skin lesion); and markers of vascular damage (hypertension, proteinuria). Arthritis was strongly associated with ICSSc. Elevated CRP, skin lesion, and pulmonary fibrosis were associated with dcSSc disease onset. Pulmonary fibrosis and elevated CRP were unlikely in IcSSc patients (Table III).

\section{Discussion}

The median age $[I Q R]$ of the disease onset in our study was $47(40 ; 52)$ years, and it was similar to data reported in EULAR and Spanish registries. But we used the first visit to a rheumatologist as the onset of the disease, whereas our European colleagues used for the same purpose the time of RP appearance. Consequently, the real age of the SSc onset may be lower and it may be approximated to the mean age in the pre-SSc group. The male/female ratio of our patients was higher than in other European countries and was comparable only to a Japanese cohort [27].

As IcSSc were classified $27.1 \%$ of patients and $72.9 \%$ - as dcSSc. These findings are in contrast to those in available to other reports [13, 14, 22]. Among all reported data, a German cohort had the most similar percentage of IcSSc and dcSSc patients (45.5\% and $32.7 \%$, respectively) [13]. This may be explained by late addressing of these patients to a rheumatologist, and it may be assumed that the most severe cases were referred to our centre. Differences in patient distribution could be also influenced by different ethnic composition of patients and the climatic conditions where they live. Almost 14\% 
Table II. Initial clinical and laboratory characteristics depending on the presence of hypertension

\begin{tabular}{|c|c|c|c|}
\hline Parameter & $\begin{array}{l}\text { Hypertension, } \\
n \text { (valid \%) }\end{array}$ & $\begin{array}{c}\text { No hypertension, } \\
n \text { (valid \%) }\end{array}$ & $p$-value \\
\hline N & $39(100)$ & $107(100)$ & \\
\hline Female & $36(92.3)$ & $103(96.3)$ & 0.38 \\
\hline Male & $3(7.7)$ & $10(3.7)$ & 0.38 \\
\hline Median age, years [IQR] & $51[47 ; 57]$ & $44[36 ; 50]$ & $<0.001$ \\
\hline IcSSc onset & $12(30.8)$ & $27(25.2)$ & 0.53 \\
\hline dcSSc onset & $27(69.2)$ & $80(74.8)$ & 0.53 \\
\hline Raynaud's phenomenon & $37(100)$ & $90(90.0)$ & 0.06 \\
\hline Skin lesion & $28(71.8)$ & $62(57.9)$ & 0.17 \\
\hline Arthritis & $34(87.2)$ & $86(80.4)$ & 0.46 \\
\hline Pulmonary fibrosis & $13(31.3)$ & $24(22.4)$ & 0.20 \\
\hline Gastrointestinal involvement & $21(72.4)$ & $56(63.6)$ & 0.38 \\
\hline Oesophagus & $19(67.8)$ & $33(49.2)$ & 0.09 \\
\hline Anaemia & $10(25.6)$ & $41(39.0)$ & 0.17 \\
\hline Abnormal leukocytes & $6(15.8)$ & $21(20.4)$ & 0.63 \\
\hline Abnormal thrombocytes & $6(25.0)$ & $19(24.1)$ & 1.0 \\
\hline $\mathrm{ESR}>25 \mathrm{~mm} / \mathrm{h}$ & $9(26.5)$ & $19(18.4)$ & 0.33 \\
\hline $\mathrm{CRP}>10 \mathrm{mg} / \mathrm{l}$ & $4(16.7)$ & $18(19.8)$ & 1.0 \\
\hline $\mathrm{RF}>14 \mathrm{IU} / \mathrm{ml}$ & $3(12.0)$ & $7(9.7)$ & 0.71 \\
\hline Proteinuria & $11(28.2)$ & $32(32.0)$ & 0.83 \\
\hline Cylindruria & $7(17.9)$ & $7(7.3)$ & 0.05 \\
\hline Mean GFR, ml/min (SD) & $77.8(28.8)$ & $91.2(20.7)$ & 0.11 \\
\hline Median PAPm, mm Hg [IQR] & $26[23 ; 35]$ & $22[16 ; 32]$ & 0.21 \\
\hline
\end{tabular}

ICSSC - limited cutaneous subset of SSC; dCSSC - diffuse cutaneous subset of SSC; ESR - erythrocyte sedimentation rate; CRP - C-reactive protein; RF-rheumatoid factor; GFR - glomerular filtration rate; PAPm - mean pulmonary arterial pressure; IQR - interquartile range; $S D$ - standard deviation

Table III. Multivariate analysis of predictors of clinical forms of systemic sclerosis

\begin{tabular}{|lccc|}
\hline Outcome & Predictor & Risk ratio & $p$ value \\
\hline IcSSc onset & Pulmonary fibrosis & 0.076 & 0.017 \\
\hline & Arthritis & 8.294 & 0.002 \\
\hline dcSSc onset & CRP $>10 \mathrm{mg} / \mathrm{l}$ & 0.204 & 0.058 \\
\hline & Skin lesion & 4.923 & 0.001 \\
\hline & Pulmonary fibrosis & 6.436 & 0.024 \\
\hline
\end{tabular}

ICSSC - limited cutaneous subset of SSc; dcSSC - diffuse cutaneous subset of SSc; CRP - C-reactive protein

of the patients of our registry were classified as pre-SSc; interestingly, it was higher, than reported in the Spanish registry (4\%) [22]. We used LeRoy and Medsger criteria for classification of these patients, but due to the absence of a clear definition of this form we excluded them from the analysis [9, 28].

In our study we observed lower frequency of pulmonary fibrosis and higher frequency of arthritis and pro- teinuria, compared to data from European registries. The prevalence of hypertension in our study was higher than in the EULAR report [14]. A similar association of IcSSc and hypertension was evident in the German registry [13]. Mean systolic pressure in the pulmonary artery in our study was lower than in the Spanish registry [22]. As we expected, clinical and laboratory signs of organ damage were revealed more often in patients of the dcSSc group. 
We revealed a strong association between age and the presence of hypertension in SSc patients. We found that hypertension occurs more frequently in IcSSc than in dcSSc patients; hypertension is also less common in pre-SSc patients. These findings fit well with data from the EULAR report, in which they claimed hypertension to be connected with "not dcSSc" and "late RP onset" [14]. In general, hypertensive patients showed higher prevalence of signs of organ damage. We found the most significant discrepancy in frequencies of signs of renal impairment. This fact could be explained by longer disease duration (because of late diagnosis of SSc) or late onset of the disease (which may be associated with poor prognosis).

Multivariate logistic regression has shown that the strongest predictive power was from signs of fibrosis and inflammation. However, the association between hypertension and clinical form of SSc, revealed in the previous section, was not confirmed. Results of this analysis fit well with reported analyses of disease manifestations [14, 22]. The only discrepancy we found was the association of arthritis with IcSSc in our study, whereas in the Spanish registry it was associated with dcSSc.

As far as we know, this is the first analysis of association between hypertension and severity of SSc. The data we gathered allow us to suggest that hypertension could be one of the pivotal signs of SSc, capable of influencing the course of the disease. The fact that hypertension is a sign that could relatively easily be revealed and controlled adds importance to our findings.

\section{Conclusions}

The most common clinical form of SSc in our study was diffuse cutaneous subset of SSc. Hypertension in patients with SSc may be associated with local cutaneous subset of SSc and renal impairment. The strongest predictors of clinical form of SSc are signs of fibrosis (skin lesion and pulmonary fibrosis) and inflammation (arthritis and elevated CRP).

\section{Limitations}

Data in this study were collected from patients living in Eastern Europe (Dnipro region, Central Ukraine).

We assumed the disease onset to be the time of the first visit to a rheumatologist, whereas other investigators used the time of Raynaud's phenomenon onset for the same purpose.

The authors declare no conflict of interest.

\section{References}

1. Roberts-Thomson P, Jones M, Hakendorf P, et al. Scleroderma in South Australia: epidemiological observations of possible pathogenic significance. Intern Med J 2001; 31: 220-229.

2. Alamanos Y, Tsifetaki N,Voulgari PV, et al. Epidemiology of systemic sclerosis in northwest Greece 1981 to 2002. Semin Arthritis Rheum 2005; 34: 714-720.

3. Nikpour M, Stevens MW, Herrick AL, et al. Epidemiology of systemic sclerosis. Best Pract Res Clin Rheumatol 2010; 74: 857869.

4. Silman AJ. Epidemiology of scleroderma. Ann Rheum Dis 1991; 50: 846-853.

5. Dobrota R, Maurer B, Graf N, et al. Prediction of improvement in skin fibrosis in diffuse cutaneous systemic sclerosis: a EUSTAR analysis. Ann Rheum Dis 2016; 75: 1743-1748.

6. Allanore $Y$, Denton CP, Krieg T, et al. Clinical characteristics and predictors of gangrene in patients with systemic sclerosis and digital ulcers in the Digital Ulcer Outcome Registry: a prospective, observational cohort. Ann Rheum Dis 2016; 75: 1736-1740.

7. Subcommittee for Scleroderma Criteria of the American Rheumatism Association Diagnostic and Therapeutic Criteria Committee. Preliminary criteria for the classification of systemic sclerosis (scleroderma). Arthritis Rheum 1980; 23: 581-590.

8. LeRoy EC, Black C, Fleischmajer R, et al. Scleroderma (systemic sclerosis): classification, subsets and pathogenesis. J Rheumatol 1988; 15: 202-205.

9. LeRoy EC, Medsger TA. Criteria for the classification of early systemic sclerosis. J Rheumatol 2001; 28: 1573-1576.

10. van den Hoogen F, Khanna D, Fransen J, et al. 2013 classification criteria for systemic sclerosis: an American College of Rheumatology/European league against rheumatism collaborative initiative. Ann Rheum Dis 2013; 72: 1747-1755.

11. Minier T, Guiducci S, Bellando-Randone S, et al. Preliminary analysis of the very early diagnosis of systemic sclerosis (VEDOSS) EUSTAR multicentre study: evidence for puffy fingers as a pivotal sign for suspicion of systemic sclerosis. Ann Rheum Dis 2014; 73: 2087-2093.

12. Kuryata OV, Lysunets TK, Semenov VV. Risk and predictors of development of arterial hypertension in patients with systemic sclerosis. Arterial Hypertension 2017; 53, doi: http://dx.doi. org/10.22141/2224-1485.3.53.2017.106849.

13. Hunzelmann N, Genth E, Krieg T, et al. The registry of the German Network for Systemic Scleroderma: frequency of disease subsets and patterns of organ involvement. Rheumatology 2008; 47: 1185-1192.

14. Walker UA, Tyndall A, Czirjak L, et al. Clinical risk assessment of organ manifestations in systemic sclerosis: a report from the EULAR cleroderma Trials And Research group database. Ann Rheum Dis 2007; 66: 754-763.

15. Agca R, Heslinga SC, Rollefstad S, et al. EULAR recommendations for cardiovascular disease risk management in patients with rheumatoid arthritis and other forms of inflammatory joint disorders: 2015/2016 update. Ann Rheum Dis 2017; 76: 17-28. 
16. Underwood JC, Cross SS. Osteoarticular and connective tissues. In: General and Systematic Pathology. Hughes DE (ed.). 5th Edition. Churchill Livingstone, Edinburgh 2009; 710-474.

17. Argula RG, Harley RA, Silver RM, et al. Is systemic sclerosis related pulmonary arterial hypertension a distinct phenotype? A lung morphometric analysis of systemic sclerosis associated vs idiopathic pulmonary arterial hypertension. J Scleroderma Relat Disord 2017; 2 (suppl 1): s1-s20.

18. Cannon PJ, Hassar M, Case DB, et al. The relalioship of hypertension and renal failure in scleroderma (progressive systemic sclerosis) to structural and functional abnormalities of the renal cortical circulation. Medicine 1974; 53: 1-46

19. Kovalchik MT, Guggenheim SJ, Silverman MH, et al. The kidney in progressive systemic sclerosis: A prospective study. Ann Intern Med 1978; 89: 881-887.

20. Simon NM, Graham MB, Kyser FA, et al. Resolution of renal failure with malignant hypertension in scleroderma: Case report and review of the literature. Am J Med 1979; 67: 533-539.

21. Kowal-Bielecka O, Fransen J, Avouac J et al. Update of EULAR recommendations for the treatment of systemic sclerosis. Ann Rheum Dis 2017; 76: 1327-1339.

22. Simeón-Aznar CP, Fonollosa-Plá V, Tolosa-Vilella C, et al. Registry of the Spanish Network for Systemic Sclerosis: Clinical Pattern According to Cutaneous Subsets and Immunological Status. Semin Arthritis Rheum 2012; 41: 789-800.

23. Mancia G, Fagard R, Narkiewicz K, et al. 2013 ESH/ESC Guidelines for the management of arterial hypertension: The Task Force for the management of arterial hypertension of the European Society of Hypertension (ESH) and of the European Society of Cardiology (ESC). Eur Heart J 2013; 34: 2159-2219.

24. Bryan C, Knight C, Black CM, et al. Prediction of five-year survival following presentation with scleroderma. Arthritis Rheum 1999; 42: 2660-2665.

25. Tent H, Waanders F, Krikken JA, et al. Performance of MDRD study and CKD-EPI equations for long-term follow-up of nondiabetic patients with chronic kidney disease. Nephrol Dial Transplant 2012; 27 Suppl 3: iii89-95.

26. Shanmugam VK, Steen VD. Renal Manifestations in Scleroderma: Evidence for Subclinical Renal Disease as a Marker of Vasculopathy. Int J Rheumatol 2010; pii: 538589.

27. Tamaki T, Mori S, Takehara K. Epidemiological study of patients with systemic sclerosis in Tokyo. Arch Dermatol Res 1991; 283: 366-371.

28. Bellando-Randone S, Guiducci S, Matucci-Cerinic M. Very early diagnosis of systemic sclerosis. Pol Arch Med Wewn 2012; 122 Suppl 1: 18-23. 